\title{
Testing the Bounds: Empirical Behavior of Target Zone Fundamentals
}

\author{
J. Isaac Miller ${ }^{1,2}$ \\ Department of Economics, University of Missouri \\ 118 Professional Building, Columbia, Missouri 65211, USA
}

\begin{abstract}
Standard target zone exchange rate models are based on nonlinear functions of an unobserved economic fundamental, which is assumed to be bounded, similarly to the target zone exchange rates themselves. A violation of this key assumption is a basic structural reason for model failure. Using a novel estimation and testing strategy, we show it is also a testable assumption. Our empirical results cast serious doubt on its validity in practice, providing a primitive reason for well-documented rejections of the basic model. Model failure from this violation is robust to otherwise ideal circumstances (e.g., perfect credibility).
\end{abstract}

First draft: February 10, 2008

Current draft: April 15, 2009

JEL Classification: F3, C5

Key words and phrases: target zone exchange rates, economic fundamental, unscented Kalman filter, rescaled range statistic

\footnotetext{
${ }^{1}$ Correspondence: millerjisaac@missouri.edu, 573-882-7282(ph), 573-882-2697(fx).

${ }^{2}$ The author is particularly grateful to Shawn Ni and Ron Ratti for several useful discussions on this topic. The author is also grateful to Marie Bessec and participants of seminars at UBC and SFU, of brown bag lunches at MU and Texas A\&M, and of the 2008 meeting of the Midwest Econometrics Group (KU) and the 2008 Missouri Economics Conference (MU) for useful feedback. The usual caveat applies.
} 


\section{Introduction}

As Sarno and Taylor (2001) emphasize, understanding how official intervention in the foreign exchange rate market works is of major policy importance. Greater comprehension of the implications of the established models of the effects of exchange rate intervention is essential in achieving this understanding. Our objective in this paper is to point out an important weakness of Krugman's (1991) prototypical target zone model, upon which many subsequent models in the literature have been built, and to highlight the need for a new paradigm in structural modeling of exchange rates that are not allowed to float.

Krugman's target zone exchange rate model posits that exchange rates are driven by a nonlinear function of an unobserved economic fundamental. ${ }^{3}$ The model has not held up well to empirical tests in the literature. In some cases, the causes for rejection (and subsequent modification) have been explicitly attributed to realignments (e.g., Bertola and Caballero, 1992; Bertola and Svensson, 1993), intramarginal interventions (e.g., Flood and Garber, 1991; Bartolini and Prati, 1999; Bessec, 2003), and other causes. However, significant failure of a more primitive assumption provides a specific reason for the well-documented difficulties of the model, even under the most ideal circumstances (e.g., no realignments).

The aim of this paper is to provide a testable structural reason for model failure, based on the implicit but critical assumption underlying the Krugman solution that the fundamental is bounded. Using a logistic functional approximation of the Krugman model (Lundbergh and Teräsvirta, 2006; Miller and Park, 2008) that is robust to violations of the assumption, and using a novel estimation and testing strategy, our empirical evidence suggests that the assumption does not generally hold. The unscented Kalman filter (Julier and Uhlmann, 1997; Julier et al., 2000) is used to estimate the unobserved series (the fundamental) from a nonlinear transformation of that series (the exchange rate). Both standard unit root tests and non-standard unit root tests robust to bounded nonlinear alternatives, for 16 fundamentals estimated from exchange rates during targeting episodes, do not support the boundedness assumption of the Krugman model, overall.

In the following section, we briefly review the Krugman target zone model and explain its vulnerability to an unbounded fundamental. We discuss robust respecification and outline our testing strategy. We present our empirical findings in Section 3 and offer some concluding remarks in Section 4.

\footnotetext{
${ }^{3}$ Nonlinearity is of course a key feature in many exchange rates models. In the target zone literature (e.g., Krugman, 1991; Flood and Garber, 1991; Bertola and Caballero, 1992; Bertola and Svensson, 1993), nonlinearity due to exchange rate intervention is critical. Nonlinearities in nominal and real exchange rates stemming from other sources are also well documented. Sarno (2003) reviews nonlinear exchange rate models. For example, transaction costs may cause nonlinearities in real exchange rates (Sercu et al., 1995; Michael et al., 1997; Taylor et al., 2001). Taylor and Peel (2000) model similar nonlinearities in nominal exchange rates. Still other authors, such as Bessec (2003), Altavilla and De Grauwe (2005), and Crespo-Cuaresma et al. (2007), use regime-switching models to introduce nonlinearity into the relationship between an exchange rate and its fundamental.
} 


\section{Target Zone Model}

A cornerstone of the well-known Krugman (1991) target zone model, which has provided the basis for many subsequent target zone models in the literature, is an economic fundamental that drives the exchange rate. This fundamental $\left(x_{t}\right)$ is defined by

$$
x_{t} \equiv m_{t}+v_{t},
$$

where $m_{t}$ represents the log of the domestic money stock, and $v_{t}$ is an all-inclusive term representing exogenous velocity shocks. In Krugman's continuous-time framework, $\left(v_{t}\right)$ is assumed to follow Brownian motion (having infinite total variation) with variance $\sigma^{2}$ and possible drift, and $\left(m_{t}\right)$ is assumed to have finite total variation. ${ }^{4}$ The discrete-time analog of such assumptions are that $\left(v_{t}\right)$ is a random walk process with drift and that $\left(m_{t}\right)$ is smoother in the sense that it either does not change every period or changes deterministically. Empirical evidence for the random walk assumption may be found in free-floating exchange rates (Meese and Rogoff, 1983). In that case, there is a linear relationship between the exchange rate and fundamental, which should be cointegrated in some sense.

In the target zone case, the monetary authorities use unsterilized interventions in the domestic money market in order to try to maintain the zone. As markets anticipate these interventions, this relationship becomes nonlinear. For this reason, Krugman and subsequent authors have postulated a nonlinear relationship

$$
s_{t}=S\left(x_{t}\right)+\varepsilon_{t},
$$

between the exchange rate and fundamental. ${ }^{5}$

A discrete-time analog of the well-known solution to the model is given by

$$
S_{0}\left(x_{t}\right)=x_{t}+\lambda \mu+B_{1} e^{\rho_{1} x_{t}}+B_{2} e^{\rho_{2} x_{t}}
$$

where $\rho_{1,2} \equiv-\mu / \sigma^{2} \pm \sqrt{2 / \lambda \sigma^{2}+\mu^{2} / \sigma^{4}}$ and $B_{1}<0, B_{2}>0$ are constants determined by setting the tangent points of the function equal to the lower and upper edges of the target zone (the so-called "smooth pasting" conditions). Specifically, these are

$$
\begin{aligned}
& B_{1}=\frac{\lambda \sigma^{2} \rho_{2}}{2}\left(\frac{\exp \left(\rho_{2} x^{-}\right)-\exp \left(\rho_{2} x^{+}\right)}{\exp \left(\rho_{1} x^{+}+\rho_{2} x^{-}\right)-\exp \left(\rho_{1} x^{-}+\rho_{2} x^{+}\right)}\right) \quad \text { and } \\
& B_{2}=\frac{\lambda \sigma^{2} \rho_{1}}{2}\left(\frac{\exp \left(\rho_{1} x^{+}\right)-\exp \left(\rho_{1} x^{1}\right)}{\exp \left(\rho_{1} x^{+}+\rho_{2} x^{-}\right)-\exp \left(\rho_{1} x^{-}+\rho_{2} x^{+}\right)}\right)
\end{aligned}
$$

where $x^{+}$and $x^{-}$are the fundamental bounds. The drift parameter $\mu$ captures not only drift in $\left(v_{t}\right)$, but also drift in $\left(m_{t}\right)$. In the absence of target zone realignments, and if the

\footnotetext{
${ }^{4} \mathrm{~A}$ process with finite total variation may be written as the difference of two increasing functions (see, e.g., Harrison, 1985, pg. 133), and is therefore smoother than that with infinite total variation. Note that finite total variation does not imply any bound on $\left(m_{t}\right)$ itself.

${ }^{5}$ The Krugman solution assumes $\left(\varepsilon_{t}\right) \equiv 0$, but the $\left(\varepsilon_{t}\right)$ is often added (Meese and Rose, 1990; Flood et al., 1990; inter alia) to allow for estimation error and idiosyncratic deviations from the target zone.
} 


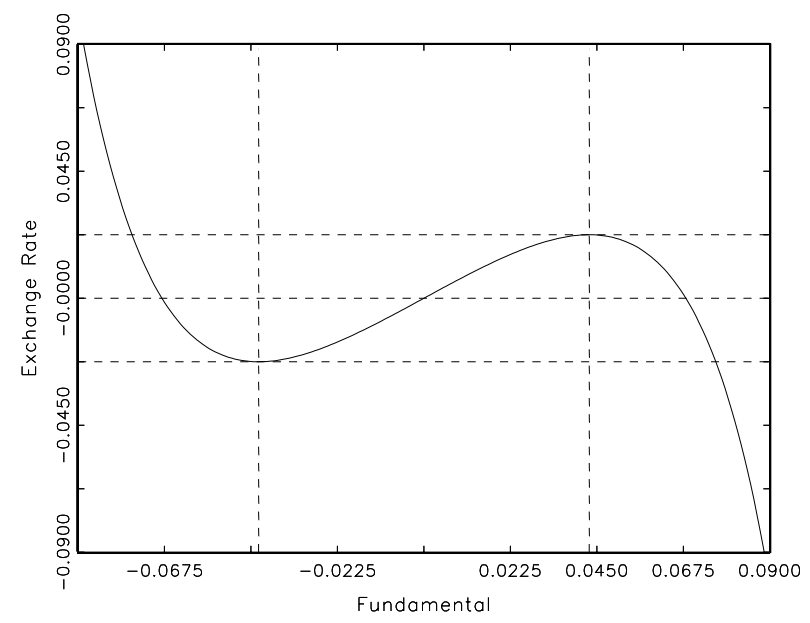

Figure 1: Krugman target zone function with explicit target zone (horizontal lines) and implicit fundamental bounds (vertical lines).

exchange rate series is transformed to be deviations from the target rate, we may expect that these drifts will roughly cancel each other out, so that $\mu$ should be small. This seems to be borne out in empirical work. Based on the interpretation of this model as a flexible price monetary model, $\lambda$ is the interest rate semi-elasticity of money demand.

\section{Model Failure from an Unbounded Fundamental}

Figure 1 illustrates the Krugman function given by (2). As long as the fundamental stays within some implicit band, delineated by the two vertical lines, the familiar "S"-shaped function maps an exchange rate within the explicit target zone, given by the three horizontal lines. (The central horizontal line in this and subsequent figures represents the exchange rate target or central parity, while the outer lines represent the edges of the target zone.) To avoid confusion, we will henceforth refer to the vertical band as the fundamental band defined by fundamental bounds $x^{+}$and $x^{-}$, while the horizontal band is the usual target zone or exchange rate band.

The fundamental bounds are critical, since the economic intuition of the model breaks down when the fundamental exceeds these bounds. Beyond them, the predicted exchange rate begins to move in the opposite direction of the fundamental. Even for moderate deviations, the predicted exchange rate deviates from the target zone in the opposite direction. Such a reversal may be conceivable in the short-run presence of some kind of bubble, but should not be an important feature of a target zone model.

The exogenous velocity shocks will exceed the fundamental bounds eventually (both by assumption and from empirical evidence). The log of the money stock must therefore increase or decrease - potentially without bound - in order to counteract velocity shocks beyond the fundamental bounds, and thus to bound both the fundamental and exchange rate. Such a requirement is a critical weakness of the Krugman and many subsequent target zone models. 

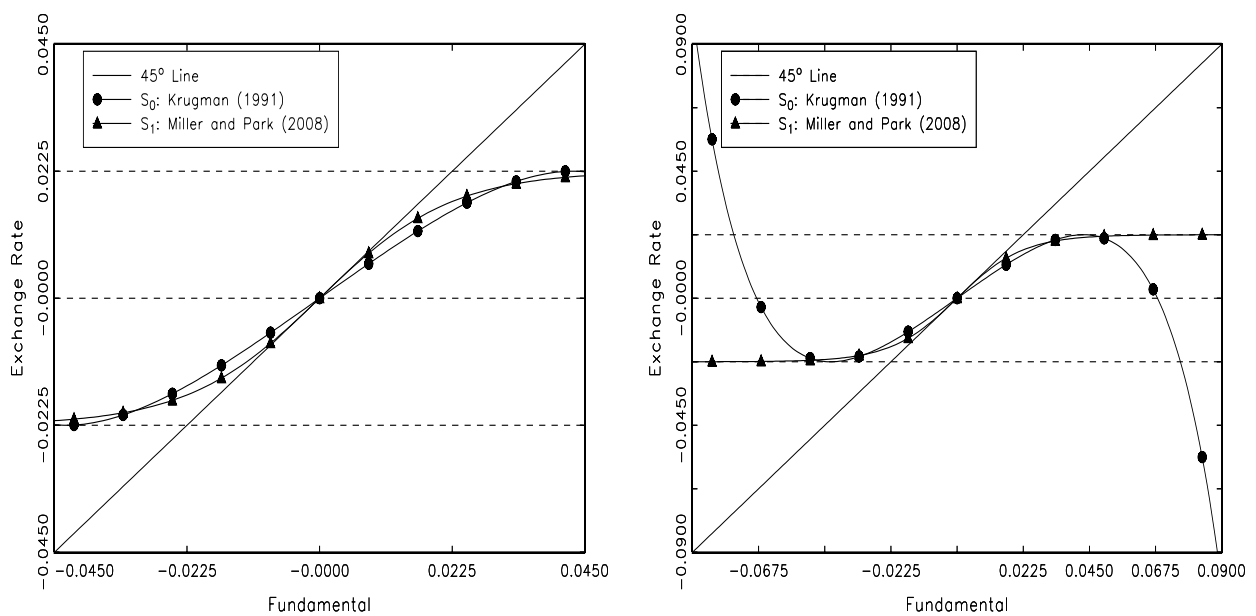

Figure 2: Comparison of $S_{0}$ and $S_{1}$ (a) within the band, and (b) beyond the band.

Moreover, as may be seen in Figure 1 and Figure 2(b), the Krugman function maps three different values of the fundamental to each exchange rate within the target zone, posing an identification problem in estimating the fundamental. In principle, enforcing the fundamental bounds may be used to identify the fundamental in estimation. However, our testing strategy precludes such enforcement.

\section{Robust Respecification}

In order to avoid model failure from a persistent fundamental and identify the fundamental for estimation and testing, a monotone increasing function may be used. Lundbergh and Teräsvirta (2006) and Miller and Park (2008) use logistic functions. The former authors use past exchange rates as the fundamental, while the latter authors estimate it. Specifically, we let

$$
S_{1}\left(x_{t}\right)=\nu-h / 2+h /\left(1+\exp \left(-\left(x_{t}-\nu\right) / \gamma\right)\right)
$$

where $\exp \nu$ is the central parity, $2(\exp h / 2-1)$ is the bandwidth, and $\gamma$ is a slope parameter. Although this function does not explicitly solve the continuous time model, it approximates the solution within the band, as illustrated in Figure 2(a), while allowing identification of the fundamental. As is clear from Figure 2(b), $S_{1}$ is robust to potentially unbounded fundamentals, since large fundamentals are still mapped inside the band. Moreover, the fundamental is identified up to the error term $\left(\varepsilon_{t}\right)$. Essentially, $S_{1}$ replaces the smooth pasting requirements used to derive $S_{0}$ with the requirement of monotonicity for identification. ${ }^{6}$

\footnotetext{
${ }^{6}$ Iannizzotto and Taylor (1999) and Taylor and Iannizzotto (2001) estimate $B_{1}, B_{2}$, and $\mu$ to be very small for BEF/DEM, FRF/DEM, and DKK/DEM exchange rates, so that the function is nearly linear within the band. A piecewise linear specification would allow identification of the fundamental for exchange rates strictly within the band, but not for those on the band. The logistic model may approximate the piecewise linear model arbitrarily well by varying the slope parameter, but allows identification.
} 
Although the Krugman model has been estimated and tested by many authors over the last two decades - de Jong (1994), Beetsma (1995), Iannizzotto and Taylor (1999), Taylor and Iannizzotto (2001), inter alia - the focus in much of this literature has been exclusively on the model parameters, with little attention paid to the fundamental itself. These authors use the method of simulated moments, which necessitates distributional assumptions that bound the simulated fundamental. In testing the bounds, we wish to avoid such assumptions.

All of our empirical results use (1) with $S$ given by $S_{1}$ in (3), estimated using the unscented Kalman filter (Julier and Uhlmann, 1997; Julier et al., 2000), a nonlinear filter that is more robust to biases inherent in the more well-known extended Kalman filter. Estimates of the fundamental are denoted by $\left(\hat{x}_{t}\right){ }^{7}$

\section{Formal Tests}

Standard linear unit root tests, such as the Phillips-Perron tests, may not have high power against bounded, nonlinear alternatives. In the specific context of target zone exchange rates, this lack of power has been noted by Taylor and Iannizzotto (2001), Taylor et al. (2001), and Cavaliere (2005) for nominal exchange rates and by Kapetanios et al. (2003) for real exchange rates. Such tests are consequently not appropriate for testing boundedness of the target zone exchange rates themselves.

However, linear unit root tests may still be appropriate for an estimated fundamental series. Since the fundamental is defined as a random walk (with possible drift) plus the log of the domestic money stock, money does not have any substantial impact on the statistical properties of the random walk under a unit root null. Since $\left(m_{t}\right)$ cannot be a random walk by Krugman's assumption, no linear combination of $\left(m_{t}\right)$ and $\left(v_{t}\right)$ - including $\left(x_{t}\right)$ - can be covariance stationary. However, $\left(m_{t}\right)$ and $\left(v_{t}\right)$ may be cointegrated in a wider sense. To the extent that $\left(m_{t}\right)$ is approximately $\mathrm{I}(1)$, a linear testing strategy for $\left(x_{t}\right)$ with $\mathrm{I}(0)$ null may be appropriate.

We construct both KPSS and Phillips-Perron $Z_{c}$ (coefficient test) and $Z_{t}$ (t-test) statistics. To the extent that the estimated fundamentals approximate the actual fundamentals, rejection of the $\mathrm{I}(0)$ null of the KPSS test can rule out approximate cointegration of $\left(m_{t}\right)$ and $\left(v_{t}\right)$. Phillips-Perron tests with $\mathrm{I}(1)$ nulls may provide evidence against the boundedness of the fundamentals.

On the other hand, since the fundamental is not directly observed, and some of our estimation techniques are explicitly nonlinear, it may be more appropriate to use a testing strategy that is robust to nonlinear, bounded alternatives. Cavaliere (2005) has used variations of the rescaled range statistic (Hurst, 1951; Mandelbrot and Wallis, 1969; Lo, 1991)

\footnotetext{
${ }^{7}$ The fundamental is often defined explicitly based on assumptions of the flexible price monetary model. See, for example, Meese and Rose (1990), Flood et al. (1990), Svensson (1991). In principle, we may use this definition to incorporate income, money stock, and other covariates directly into the model. Such an approach has at least two serious drawbacks: (a) Lower frequency data for these series lead to very small sample sizes, and (b) assumptions about purchasing power parity and uncovered interest parity must be addressed. Pursuing this approach for the first five exchange rates below (with time periods over which sufficiently monthly data are available) yielded qualitatively similar test results to those reported below.
} 
to test boundedness of the exchange rates themselves. Similarly to Cavaliere (2005), we use

$$
\begin{aligned}
\hat{R}_{n} & \equiv n^{-1 / 2}\left(\max _{t=1, \ldots, n}\left(\hat{x}_{t}\right)-\min _{t=1, \ldots, n}\left(\hat{x}_{t}\right)\right) / \hat{\omega}_{n} \\
\hat{L}_{n} & \equiv-n^{-1 / 2} \min _{t=1, \ldots, n}\left(\hat{x}_{t}-\bar{x}_{n}\right) / \hat{\omega}_{n} \\
\hat{U}_{n} & \equiv n^{-1 / 2} \max _{t=1, \ldots, n}\left(\hat{x}_{t}-\bar{x}_{n}\right) / \hat{\omega}_{n}
\end{aligned}
$$

where $\bar{x}_{n}$ is the sample mean of $\hat{x}_{t}$, and $\hat{\omega}_{n}$ is a consistent estimator of the long-run variance of $n^{-1 / 2} \sum_{t=1}^{n} \triangle \hat{x}_{t}{ }^{8}$ Under the null, $\left(\hat{x}_{t}\right) \sim I(1)$. If the de facto bounds of the data, given by the range and the upper and lower bounds, increase with the sample size, these tests point to the absence of bounds. If they do not increase with the sample size, then the series may be bounded.

All of our tests are conducted on estimated fundamentals, rather than actual unobserved fundamentals, and should be interpreted appropriately. Constructing tests of the actual fundamentals based on these estimates would be much more complicated, requiring nonstandard critical values, and may in fact be infeasible.

\section{Plausible Interest Rate Semi-Elasticity of Money Demand?}

As an additional check, we look at the implied interest rate semi-elasticity of money $\tilde{\lambda}$ of the model. Any series of observations of real economic activity is bounded in finite samples. The range tests above simply tell us whether or not the de facto bounds given by the range of the data are increasing with the sample size. Even if these bounds do not increase (and we reject the null), the bounds may be unrealistically large.

How wide can the fundamental band plausibly be? Using $\tilde{x}_{t}=\hat{x}_{t}-x^{*}$, where $x^{*}$ is the exchange rate target, we calculate an empirical band to estimate $x^{+}$. We use the largest absolute deviation of the estimated fundamental from the central parity (restricting $\mu=0$ ), unless this does not exceed the announced exchange rate band. We then estimate the longrun variance of $\left(\triangle \tilde{x}_{t}\right)$, which approximates $\sigma^{2}$ in discrete time if $\left(\hat{x}_{t}\right)$ never exceeds its band. We can then identify (numerically) the elasticity $\tilde{\lambda}$ that generates the known bands on the exchange rate. If this elasticity is implausible, then the empirical range of the fundamental cannot realistically lie within the fundamental band of the model.

If $\left(\tilde{x}_{t}\right)$ does not exceed the exchange rate band, then $\lambda \rightarrow 0$, which would be the case if either markets do not think the band is credible and so do not smooth the function very much, or if the estimated fundamental does not wander far from the central parity.

\section{Data and Empirical Results}

Our empirical results focus on ten exchange rate mechanism (ERM) I exchange rates, five ERM II exchange rates, and one U.S. dollar peg. Many empirical estimates of the Krugman model - whether favorable or unfavorable - have used data from the ERM I period. The European financial crisis of the early 1990's resulted in a widening of the target zones for

\footnotetext{
${ }^{8}$ We use the quadratic spectral kernel with data-driven bandwidth selection based on Andrews (1991) for long-run variance estimation.
} 


\begin{tabular}{|l|lr|rr|}
\hline & \multicolumn{1}{|c|}{ Dates } & Band $( \pm)$ & Dates & Band $( \pm)$ \\
\hline Belgian Franc (BEF/DEM) & $04 / 02 / 91-08 / 02 / 93$ & $2.25 \%$ & $08 / 03 / 93-12 / 31 / 98$ & $15 \%$ \\
French Franc (FRF/DEM) & $04 / 02 / 91-08 / 02 / 93$ & $2.25 \%$ & $08 / 03 / 93-12 / 31 / 98$ & $15 \%$ \\
Dutch Guilder (NLG/DEM) & $04 / 02 / 91-12 / 31 / 98$ & $2.25 \%$ & & \\
Danish Krone (DKK/DEM) & $01 / 04 / 91-08 / 02 / 93$ & $2.25 \%$ & $08 / 03 / 93-12 / 31 / 98$ & $15 \%$ \\
Danish Krone (DKK/EUR) & $01 / 04 / 99-09 / 28 / 07$ & $2.25 \%$ & & \\
Irish Pound (IEP/DEM) & $08 / 02 / 93-12 / 31 / 98$ & $15 \%$ & & \\
Aus. Schilling (ATS/DEM) & $01 / 09 / 95-12 / 31 / 98$ & $15 \%$ & & \\
Spanish Peseta (ESP/DEM) & $03 / 06 / 95-12 / 31 / 98$ & $15 \%$ & & \\
Portug. Escudo (PTE/DEM) & $03 / 06 / 95-12 / 31 / 98$ & $15 \%$ & & \\
Finnish Markka (FIM/DEM) & $10 / 14 / 96-12 / 31 / 98$ & $15 \%$ & & \\
Italian Lira (ITL/DEM) & $11 / 25 / 96-12 / 31 / 98$ & $15 \%$ & & \\
Greek Drachma (GRD/EUR) & $01 / 04 / 99-12 / 29 / 00$ & $15 \%$ & & \\
Slovenian Tolar (SIT/EUR) & $06 / 28 / 04-12 / 29 / 06$ & $15 \%$ & \\
Cyprus Pound (CYP/EUR) & $05 / 02 / 05-12 / 31 / 07$ & $15 \%$ & \\
Latvian Lats (LVL/EUR) & $05 / 02 / 05-12 / 31 / 07$ & $1 \%$ & \\
Saudi Riyal (SAR/USD) & $08 / 24 / 98-12 / 31 / 07$ & $1 \%$ & \\
\hline
\end{tabular}

Table 1: Exchange rates in the sample, time periods, and respective bandwidths.

many ERM I rates. Especially volatile fundamentals that exceeded the theoretical fundamental bounds may be estimated during this period, even though no realignments occurred. Our tests may therefore shed light on some of the rejections of the Krugman model. ERM II exchange rates provide insights into more contemporary target zone arrangements, since several EU members are still bound by the ERM II, as of this writing. Saudi Arabia has pegged the Riyal to the U.S. dollar, unofficially since 1986 and officially since 2003. The Riyal is allowed to fluctuate within a narrow $\pm 1 \%$ band around this peg. Maintaining the peg became a controversial issue for Saudi Arabia in 2007, as a weak dollar drove other oil-exporting countries to drop their pegs.

We analyze the exchange rates in Table 1 . The beginnings of most series coincide either with the last realignment of the respective central rates or with entrance of the country into the ERM. The central rate for the GRD/EUR rate was realigned roughly halfway through its two-year target zone period. Since this realignment was slight relative to the bandwidth of the zone and the actual fluctuations of the exchange rate, we do not model it explicitly.

In order to estimate the fundamental for the three rates whose bands were widened, we explicitly model the (known) change in the bandwidth and allow for a contemporaneous structural break in estimation of the (unknown) slope parameter $\gamma$.

We obtained European data from EuroStat and Saudi data from Pacific Exchange Rate Service (University of British Columbia).

\section{Estimated Fundamentals}

Figure 3 shows the exchange rates and estimated fundamentals. The fundamentals exhibit stochastic trends, straying from their mean when the respective exchange rates near the edge of their band. Since any potential fundamental bounds do not coincide with the bounds impose on the exchange rate, boundedness of these stochastic trends is an open question. Note that most of the ERM I and ERM II exchange rates and fundamentals 

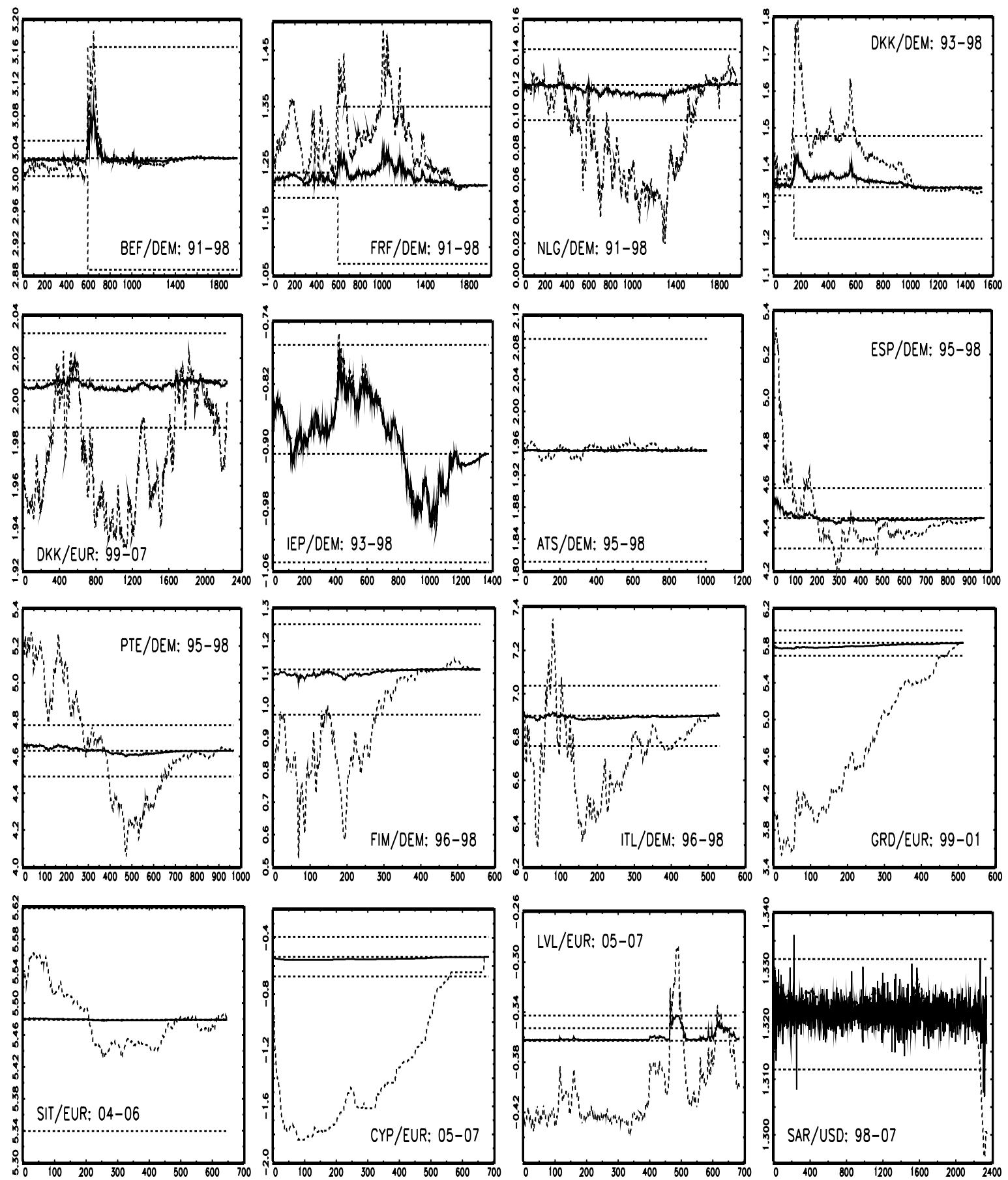

Figure 3: Target zone exchange rates (solid lines) and estimated fundamentals (dashed lines). Horizontal lines represent the target and target zone (not the fundamental band). 
appear to converge to their targets leading up to the adoption of the Euro. ${ }^{9}$ Interestingly, the DKK/DEM rate also shows convergence even though Denmark did not adopt the Euro. The last two exchange rates, which were sampled through 2007, show recent (as of this writing) pressure to move outside of their respective bands. Clearly, Latvia has struggled throughout this period against pressure to appreciate the Lats. Saudi Arabia began to face similar pressure to maintain its peg to the dollar in the face of U.S. rate cuts. These pressures may not be sustainable in the long run, and Saudi Arabia may drop the peg, as Kuwait did in 2007.

\section{Test Results and Implied Elasticity}

Test results are shown in Table 2, with significance at the $5 \%$ level is indicated. The KPSS fails to reject the $\mathrm{I}(0)$ null for most of the exchange rates, but rejects for half of the fundamentals, suggesting that the exchange rates themselves may be bounded, but the fundamentals are generally not bounded. Moreover, the unit root null cannot be rejected using the Z-tests for any fundamentals except those driving the Schilling and Peseta. The evidence is much more mixed using the range-based tests, since some fundamentals have significantly larger empirical minima than maxima or vice versa. Based on these, we find evidence that Schilling fundamental was bounded, the Krone fundamental may have been bounded since 1998, and weaker evidence about the French Franc fundamental.

Overall, the evidence strongly points towards a bounded fundamental for the Schilling, and unbounded fundamentals for the Irish Pound, Escudo, Drachma, Tolar, and Cyprus Pound. The evidence more weakly points to unbounded fundamentals for the Markka and for the Krone before 1999. Evidence for the remaining rates is more mixed.

It is also instructive to look at the differences between the tests on the estimated fundamentals and those on the exchange rates themselves. The most striking are the ITL/DEM and SAR/USD rates. For both of these, the evidence strongly favors bounded exchange rates but much less so for the estimated fundamentals.

Table 2 also presents implied elasticities calculated using the strategy discussed above. Specifically, we calculate the elasticities $\tilde{\lambda}$ that yield the correct exchange rate band, ${ }^{10}$ given the empirical variance and deviation from the target rate of the estimated fundamental $\left(\hat{x}_{t}\right)$. Most of these elasticities are obviously implausible. The most plausible elasticities - for the ATS/DEM and SIT/EUR rates - correspond to rates that seem to adhere to a much stricter

\footnotetext{
${ }^{9}$ As von Hagen and Traistaru (2005) noted, markets reacted to expected convergence of the exchange rates to their central parities as early as September 1997. A possible way to model this might be a gradual narrowing of the implicit band described by the function - i.e., a time-varying band parameter $h_{t}-$ or by a gradual tightening of expectations around the parity - i.e., a time-varying slope parameter $\gamma_{t}$.

Another strategy to deal with this convergence would be to simply drop the data from this period. However, we may also expect that the fundamental itself may be more tightly regulated during this period. Dropping these data may bias our results against the Krugman model. By including these data but not explicitly modeling the changing expectations, we may expect our results to be biased (if at all) in favor of the Krugman model, since our filtering strategy will generate tightly bounded filtered fundamentals corresponding to exchange rates near the central parity.

${ }^{10}$ For BEF/DEM, FRF/DEM, and DKK/DEM, which experienced a one-time widening of the band to $\pm 15 \%$, we use $\pm 15 \%$ for the whole sample.
} 


\begin{tabular}{|c|c|c|c|c|c|c|c|c|c|c|c|c|c|}
\hline & \multicolumn{6}{|c|}{$\left(s_{t}\right)$} & \multicolumn{6}{|c|}{ Estimated $\left(\mathrm{x}_{, \mathrm{t}}\right)$} & \multirow{2}{*}{$\begin{array}{c}\text { Est } \\
\lambda\end{array}$} \\
\hline & KPSS & $Z_{c}$ & $Z_{t}$ & $\mathrm{R}_{\mathrm{n}}$ & $\mathrm{L}_{\mathrm{n}}$ & $\mathrm{U}_{\mathrm{n}}$ & KPSS & $Z_{c}$ & $Z_{t}$ & $\mathrm{R}_{\mathrm{n}}$ & $\mathrm{L}_{\mathrm{n}}$ & $\mathrm{U}_{\mathrm{n}}$ & \\
\hline BEF/DEM 04/02/91-12/31/98 & 0.09 & $-20.86 * *$ & $-3.12 * *$ & 1.35 & $0.13^{* *}$ & 1.22 & 0.44 & -5.58 & -2.86 & 1.50 & $0.21 * *$ & 1.29 & 130 \\
\hline FRF/DEM 04/02/91-12/31/98 & 0.16 & $-14.20 * *$ & -2.64 & $0.91 * *$ & $0.24 *$ & 0.68 & 0.34 & -3.92 & -2.56 & $0.88 * *$ & $0.25 * *$ & 0.63 & 893 \\
\hline NLG/DEM 04/02/91-12/31/98 & 0.11 & -9.29 & -2.23 & $0.96 * *$ & 0.57 & $0.39 * *$ & 0.43 & -3.30 & -1.95 & 1.07 & 0.63 & $0.44 * *$ & 3,819 \\
\hline DKK/DEM 01/04/93-12/31/98 & 0.30 & -8.97 & -1.92 & 1.28 & $0.26 * *$ & 1.02 & $1.03 * *$ & -1.34 & -2.10 & 1.19 & $0.24 *$ & 0.95 & 4,712 \\
\hline DKK/EUR 01/04/99-09/28/07 & 0.15 & -9.86 & -2.35 & $0.78 * *$ & $0.37 * *$ & $0.41 * *$ & $0.57 * *$ & -3.35 & -2.32 & $0.76 * *$ & $0.37 * *$ & $0.39 * *$ & 1,744 \\
\hline IEP/DEM $\quad 08 / 02 / 93-12 / 31 / 98$ & 0.30 & -4.48 & -1.42 & 1.57 & 0.77 & 0.79 & $1.58 * *$ & -1.13 & -1.50 & 1.57 & 0.71 & 0.86 & 33 \\
\hline ATS/DEM 01/09/95-12/31/98 & 0.26 & $-37.45 * *$ & -2.80 & 0.97 & 0.49 & 0.49 & 0.37 & -3.73 & $-2.91 * *$ & $0.76 * *$ & $0.44 *$ & $0.32 * *$ & $\rightarrow 0$ \\
\hline ESP/DEM 03/06/95-12/31/98 & 0.26 & $-15.88 * *$ & $-3.79 * *$ & 1.77 & $0.40 * *$ & 1.36 & 0.25 & -13.34 & $-5.23 * *$ & 2.02 & $0.42 * *$ & 1.59 & 9,967 \\
\hline PTE/DEM 03/06/ & 0.25 & -5.66 & -1.92 & 1.85 & 0.91 & 0.94 & $0.47 * *$ & -2.72 & -1.97 & 1.89 & 0.93 & 0.96 & 2,570 \\
\hline FIM/DEM $\quad 10 / 14 / 96-12 / 31 / 98$ & 0.41 & -14.09 & -2.23 & 1.12 & 0.84 & $0.28 * *$ & $0.47 * *$ & -2.47 & -1.64 & 1.28 & 0.91 & $0.37 * *$ & 1,616 \\
\hline ITL/DEM 11/25/96-12/31/98 & 0.17 & $-20.44 * *$ & $-3.03 * *$ & 1.03 & $0.44 *$ & 0.59 & 0.22 & -5.93 & -2.70 & 1.07 & $0.44 *$ & 0.62 & 469 \\
\hline GRD/EUR 01/04/99-12/29/00 & $279.61 * *$ & 0.04 & 0.14 & 2.55 & 1.34 & 1.21 & $6.58 * *$ & 0.37 & -0.13 & 2.69 & 1.43 & 1.26 & 48,024 \\
\hline SIT/EUR $\quad 06 / 28 / 04-12 / 29 / 06$ & 0.23 & -10.20 & -1.61 & 1.57 & 0.60 & 0.97 & $0.97 * *$ & -1.57 & -1.54 & 1.85 & 0.69 & 1.16 & $\rightarrow 0$ \\
\hline CYP/EUR 05/02/05-12/31/07 & $3.07 * *$ & 0.63 & 0.20 & 2.43 & 0.98 & 1.45 & $1.60 * *$ & 0.96 & -0.13 & 1.82 & 0.74 & 1.08 & 25,298 \\
\hline LVL/EUR 05/02/05-12/31/07 & 0.36 & -11.83 & -2.50 & $0.89 * *$ & $0.12 * *$ & 0.77 & 0.30 & -5.02 & -2.06 & 1.24 & $0.29 * *$ & 0.95 & 1,539 \\
\hline SAR/USD 08/24/98-12/31/07 & $2.29 * *$ & $-1971 * *$ & $-9.02 * *$ & $0.76 * *$ & $0.39 * *$ & $0.37 * *$ & 0.40 & 12.98 & 0.04 & 1.47 & 1.22 & $0.25 * *$ & 17,673 \\
\hline
\end{tabular}


band than the announced exchange rate band. Even for estimated fundamentals that barely differ from the exchange rate, such as the IEP/DEM rate, the implied elasticity is large.

If there is a fundamental band with upper edge given by $x^{+}$, then it must be smaller than the empirical bands of $\left(\hat{x}_{t}\right)$ to generate plausible elasticities. In that sense, these estimated fundamentals do not support the boundedness assumption of the Krugman model.

\section{Concluding Remarks}

The doubt created by the test results and implied elasticities seriously calls into question the fundamental bounds, a key assumption of the Krugman and many subsequent target zone models. Our findings support the general skepticism that the Krugman model has already faced in the literature. Moreover, these results offer a specific explanation for the negative conclusions.

A new paradigm for structural modeling of target zone exchange rates is needed. While reduced form approaches in the literature, represented for example by contributions by Bessec (2003), Crespo-Cuaresma, et al. (2005), Lundbergh and Teräsvirta (2006), and others, provide greater modeling flexibility by requiring only lagged exchange rate data, they do not allow for inference about some of the structural macroeconomic parameters of interest.

A recent approach by Bauer et al. (2009), building on work by Frankel and Froot (1986), Brock and Hommes (1998), and De Grauwe and Grimaldi (2005, 2006), using a heterogeneous agent structure, shows promise in the direction of developing an alternative approach to the modeling of target zone exchange rates. They derive a model in which the fundamental is driven by a nonlinear stochastic differential equation, which they show through simulations may generate exchange rate behavior more consistent with target zone exchange rate data.

\section{References}

Altavilla, C. and P. De Grauwe (2005). "Non-linearities in the Relation between the Exchange Rate and Its Fundamentals," CESifo Working Paper 1561.

Andrews, D.W.K. (1991). "Heteroskedasticity and Autocorrelation Consistent Covariance Matrix Estimation," Econometrica, 59, 817-58.

Bartolini, L. and A. Prati (1999) "Soft Exchange Rate Bands and Speculative Attacks: Theory and Evidence from the ERM since August 1993," Journal of International Economics, 49, 1-29.

Bauer, C., P. de Grauwe, S. Reitz (2009). "Exchange Rate Dynamics in a Target Zone: A Heterogeneous Expectations Approach." Journal of Economic Dynamics and Control, $33,329-44$.

Beetsma, R.M.W.J. (1995). "EMS Exchange Rate Bands: A Monte Carlo Investigation of Three Target Zone Models," Journal of International Money and Finance, 14, 311-28. 
Bertola, G. and R.J. Caballero (1992). "Target Zones and Realignments," American Economic Review, 82, 520-36.

Bertola, G. and L.E.O. Svensson (1993). "Stochastic Devaluation Risk and the Empirical Fit of Target-Zone Models," Review of Economic Studies, 60, 689-712.

Bessec, M. (2003). "Mean-Reversion Versus Adjustment to PPP: The Two Regimes of Exchange Rate Dynamics Under the EMS, 1979-1998," Economic Modelling, 20, 14164.

Brock, W., and C. Hommes (1998). "Heterogeneous beliefs and routes to chaos in a simple asset pricing model," Journal of Economic Dynamics and Control, 22, 1235-74.

Cavaliere, G. (2005). "Testing Mean Reversion in Target-Zone Exchange Rates," Applied Economics, 37, 2335-47.

Crespo-Cuaresma, J., B. Égert, and R. MacDonald (2005). "Non-linear Exchange Rate Dynamics in Target Zones: A Bumpy Road towards a Honeymoon: Some Evidence from the ERM, ERM2, and Selected New EU Member States," CESifo working paper 1511.

De Grauwe, P., and M. Grimaldi (2005). "Heterogeneity of Agents, Transactions Costs and the Exchange Rate," Journal of Economic Dynamics and Control, 29, 691-719.

De Grauwe, P., and M. Grimaldi (2006). "Exchange Rate Puzzles: A Tale of Switching Attractors," European Economic Review, 50,1-33.

de Jong, F. (1994). "A Univariate Analysis of EMS Exchange Rates Using a Target Zone Model," Journal of Applied Econometrics, 9, 31-45.

Flood, R.P., Rose, A.K., and Mathieson (1990). "An Empirical Exploration of Exchange Rate Target-Zones," NBER working paper 3543.

Flood, R.P and P.M. Garber (1991). "The Linkage between Speculative Attack and Target Zone Models of Exchange Rates," The Quarterly Journal of Economics, 106, 1367-72.

Frankel, J., and K. Froot (1986). "Understanding the US Dollar in the Eighties: The Expectations of Chartists and Fundamentalists," Economic Record, 62, 24-38.

Harrison, J.M. (1985). Brownian Motion and Stochastic Flow Systems. New York: Wiley.

Hurst, H.E. (1951). "Long-term Storage Capacity of Reservoirs," Transactions of the American Society of Civil Engineers, 116, 770-99.

Iannizzotto, M. and M.P. Taylor (1999). "The Target Zone Model, Non-Linearity and Mean Reversion: Is the Honeymoon Really Over?" The Economic Journal, 109, C96C110. 
Julier, S.J. and J.K. Uhlmann (1997). "A New Extension of the Kalman Filter to Nonlinear Systems," In The Proceedings of AeroSense: The 11th International Symposium on Aerospace/Defense Sensing, Simulation and Controls, SPIE.

Julier, S.J., J.K. Uhlmann, and H.F. Durrant-Whyte (2000). "A New Method for the Nonlinear Transformation of Means and Covariances in Filters and Estimators," IEEE Transactions on Automatic Control, 45, 477-82.

Kapetanios, G., Y. Shin, and A. Snell (2003). "Testing for a Unit Root in the Nonlinear STAR Framework," Journal of Econometrics, Journal of Econometrics, 112, 359-79.

Krugman, P.R. (1991). "Target Zones and Exchange Rate Dynamics," The Quarterly Journal of Economics, 106, 669-82.

Kwiatkowski, D., P.C.B. Phillips, P. Schmidt, and Y. Shin (1992), "Testing the Null Hypothesis of Stationarity against the Alternative of a Unit Root," Journal of Econometrics, 54, 159-78.

Lo, A.W. (1991). "Long-term Memory in Stock Market Prices," Econometrica, 59, 1279313.

Lundbergh, S. and T. Teräsvirta (2006). "A Time Series Model for an Exchange Rate in a Target Zone with Applications," Journal of Econometrics, 131, 579-609.

Mandelbrot, B.B. and J.R. Wallis (1969). "Some Long-Run Properties of Geophysical Records," Water Resources Research, 5, 321-40.

Meese, R.A. and K. Rogoff (1983). "Empirical Exchange Rate Models of the Seventies: Do They Fit Out of Sample?" Journal of International Economics,14, 3-24.

Meese, R.A. and A.K. Rose (1990). "Nonlinear, Nonparametric, Nonessential Exchange Rate Estimation," The American Economic Review, 80, 192-96.

Michael, P., A.R. Nobay and D.A. Peel (1997). "Transactions Costs and Nonlinear Adjustment in Real Exchange Rates: An Empirical Investigation," The Journal of Political Economy, 105, 862-79.

Miller, J.I. and J.Y. Park (2008). "Nonlinearity, Nonstationarity, and Thick Tails: How They Interact to Generate Persistency in Memory," Working Paper 08-01, Department of Economics, University of Missouri.

Phillips, P.C.B, and P. Perron (1988). "Testing for a Unit Root in Time Series Regressions," Biometrika, 75, 335-46.

Sarno, L. (2003). "Nonlinear Exchange Rate Models: A Selective Overview," IMF Working Paper WP/03/111.

Sarno, L., and M.P. Taylor (2001). "Official Intervention in the Foreign Exchange Market: Is It Effective And, If So, How Does It Work?" Journal of Economic Literature, 39, 839-68. 
Sercu, P. Uppal, R. and C. van Hulle (1995), "The Exchange Rate in the Presence of Transactions Costs: Implication for Tests of Purchasing Power Parity," Journal of Finance, 50, 1309-19.

Svensson, L.E.O. (1991). "The Term Structure of Interest Rate Differentials in a Target Zone: Theory and Swedish Data," Journal of Monetary Economics, 28, 87-116.

Taylor, M.P. and M. Iannizzotto (2001). "On the Mean-reverting Properties of Target Zone Exchange Rates: A Cautionary Note," Economics Letters, 71, 177-29.

Taylor, M.P. and D.A. Peel (2000). "Nonlinear Adjustment, Long-run Equilibrium and Exchange Rate Fundamentals," Journal of International Money and Finance, 19, 3353.

Taylor, M.P., D.A. Peel, L. Sarno (2001). "Nonlinear Mean-Reversion in Real Exchange Rates: Toward a Solution to the Purchasing Power Parity Puzzles," International Economic Review, 42, 1015-42.

von Hagen, J. and I. Traistaru (2005). "Macroeconomic Adjustment in the New EU Member States," Working Paper B-01-2005, Center for European Integration Studies, University of Bonn. 


\section{Appendix: Unscented Kalman Filter}

\section{*** Appendix is not intended for publication. ***}

The general filtering problem may be illustrated by considering a simple generalization of our model

$$
z_{t}=g\left(x_{t}\right)+u_{t}
$$

where $\left(u_{t}^{\prime}, \triangle x_{t}\right)^{\prime}$ is an iid normal sequence with variance given by a matrix with diagonal blocks of $\Lambda$ and $\tau^{2}$ and off-diagonal blocks of zeros. The series $\left(x_{t}\right)$ is simply a latent univariate random walk. The model is characterized by unknown parameters $\Lambda, \tau^{2}$, and any unknown parameters in the possibly vector-valued and possibly nonlinear function $g$. Since the series $\left(x_{t}\right)$ is latent, a filter attempts to estimate $\left(x_{t}\right)$ based on its conditional distributions.

Define

$$
z_{t \mid \bullet} \equiv \mathbf{E} z_{t} \mid \mathcal{F}_{\bullet} \quad \text { and } \quad x_{t \mid \bullet} \equiv \mathbf{E} x_{t} \mid \mathcal{F}_{\bullet}
$$

to be conditional means, and

$$
\omega_{t \mid \bullet} \equiv \operatorname{var}\left(x_{t} \mid \mathcal{F}_{\bullet}\right), \quad \Sigma_{t \mid \bullet} \equiv \operatorname{var}\left(z_{t} \mid \mathcal{F}_{\bullet}\right), \quad \text { and } \quad \Xi_{t \mid \bullet} \equiv \operatorname{cov}\left(x_{t}, z_{t} \mid \mathcal{F}_{\bullet}\right)
$$

to be conditional variances and covariance.

Filtering generally proceeds in three steps: $[\mathrm{P}]$ one-step-ahead prediction of the conditional density of $x_{t}$, [L] likelihood calculation necessary for ML estimation and also for updating, and $[\mathrm{U}]$ updating the prediction with newly available information.

[P] Prediction. One-step-ahead prediction of the conditional density of $x_{t}$ is given by

$$
p\left(x_{t} \mid \mathcal{F}_{t-1}\right)=p\left(x_{t-1}+\triangle x_{t} \mid \mathcal{F}_{t-1}\right)
$$

in this case. Since $\left(\triangle x_{t}\right)$ is iid normal, its distribution is fully characterized by its first two moments. The prediction step may be written simply as

$$
\begin{aligned}
x_{t \mid t-1} & =x_{t-1 \mid t-1} \\
\omega_{t \mid t-1} & =\omega_{t-1 \mid t-1}+\tau^{2}
\end{aligned}
$$

in this light.

[L] Likelihood Calculation. The conditional distribution of $z_{t}$ is given by

$$
p\left(z_{t} \mid \mathcal{F}_{t-1}\right)=p\left(g\left(x_{t}\right)+u_{t} \mid \mathcal{F}_{t-1}\right)
$$

and calculating the condition likelihood function requires only the first two moments

$$
\begin{aligned}
z_{t \mid t-1} & =\mathbf{E} g\left(x_{t}\right) \mid \mathcal{F}_{t-1} \\
\Sigma_{t \mid t-1} & =\operatorname{var}\left(g\left(x_{t}\right) \mid \mathcal{F}_{t-1}\right)+\Lambda
\end{aligned}
$$

since $\left(u_{t}\right)$ is iid normal. 
[U] Updating. Updating frequently uses linear projections to update the predictions with new information available at time $t$, embodied by $\left(z_{t}-z_{t \mid t-1}\right)$. Specifically, the updating equations are

$$
\begin{aligned}
& x_{t \mid t}=x_{t \mid t-1}+K_{t \mid t-1}\left(z_{t}-z_{t \mid t-1}\right) \\
& \omega_{t \mid t}=\omega_{t \mid t-1}-K_{t \mid t-1} \Sigma_{t \mid t-1} K_{t \mid t-1}^{\prime}
\end{aligned}
$$

where

$$
K_{t \mid t-1} \equiv \Xi_{t \mid t-1} \Sigma_{t \mid t-1}^{-1}
$$

is the Kalman gain.

\section{Kalman Filter}

When $g$ is linear, say $g(x)=\gamma x$, the Kalman filter is typically employed. It is easy to see that

$$
\begin{aligned}
\mathbf{E} g\left(x_{t}\right) \mid \mathcal{F}_{t-1} & =\gamma x_{t \mid t-1} \\
\operatorname{var}\left(g\left(x_{t}\right) \mid \mathcal{F}_{t-1}\right) & =\gamma \omega_{t \mid t-1} \gamma^{\prime} \\
\operatorname{cov}\left(x_{t}, z_{t} \mid \mathcal{F}_{t-1}\right) & =\omega_{t \mid t-1} \gamma^{\prime}
\end{aligned}
$$

and the Kalman gain is thus simply

$$
\omega_{t \mid t-1} \gamma^{\prime}\left(\gamma \omega_{t \mid t-1} \gamma^{\prime}+\Lambda\right)^{-1}
$$

in this case.

\section{Unscented Kalman Filter (UKF)}

The unscented Kalman filter (Julier and Uhlmann, 1997; Julier et al., 2000) is one of many alternatives that may be used in the more general context of nonlinear $g$. This filter estimates the mean and variance of the conditional distribution of $x_{t}$ by parsimoniously choosing weighted sigma-points. Estimation works in three similar steps. For the univariate latent random walk considered here, these may be summarized as follows.

[P] Prediction. A set of three sigma points $\xi_{t-1, i}$ for $i=0,1,2$, given by

$$
\begin{aligned}
& \xi_{t-1,0}=x_{t-1 \mid t-1} \\
& \xi_{t-1,1}=x_{t-1 \mid t-1}+\sqrt{(1+\theta) \omega_{t-1 \mid t-1}} \\
& \xi_{t-1,2}=x_{t-1 \mid t-1}-\sqrt{(1+\theta) \omega_{t-1 \mid t-1}}
\end{aligned}
$$

where $\theta$ is a tuning parameter, are chosen. Then, using

$$
\xi_{t, i}=\xi_{t-1, i}
$$


the prediction equations are given by

$$
\begin{aligned}
x_{t \mid t-1} & =\sum_{i=0}^{2} w_{i} \xi_{t, i} \\
\omega_{t \mid t-1} & =\sum_{i=0}^{2} w_{i}\left(\xi_{t, i}-x_{t \mid t-1}\right)\left(\xi_{t, i}-x_{t \mid t-1}\right)^{\prime}+\tau^{2}
\end{aligned}
$$

where

$$
w_{0}=\frac{\theta}{(1+\theta)} \quad \text { and } \quad w_{i}=\frac{1}{2(1+\theta)} \quad \text { for } i=1,2
$$

are weights given to the sigma points. ${ }^{11}$

[L] Likelihood Calculation. These points are then propagated through the nonlinear function $g$, so that

$$
\begin{aligned}
\zeta_{t, i} & =g\left(\xi_{t, i}\right) \\
y_{t \mid t-1} & =\sum_{i=0}^{2} w_{i} \zeta_{t, i} \\
\Sigma_{t \mid t-1} & =\sum_{i=0}^{2} w_{i}\left(\zeta_{t, i}-z_{t \mid t-1}\right)\left(\zeta_{t, i}-z_{t \mid t-1}\right)^{\prime}+\Lambda
\end{aligned}
$$

are used to calculate the conditional likelihood.

[U] Updating. Updating proceeds in exactly the same way as in the linear case, with

$$
K_{t \mid t-1}=\sum_{i=0}^{2} w_{i}\left(\xi_{t, i}-x_{t \mid t-1}\right)\left(\zeta_{t, i}-z_{t \mid t-1}\right)^{\prime} \Sigma_{t \mid t-1}^{-1}
$$

as the Kalman gain.

\footnotetext{
${ }^{11}$ Subsequent authors have refined the UKF by incorporating additional tuning parameters. Following Julier et al. (2000), we set $\theta=2$ (since $\left(x_{t}\right)$ is univariate) and do not incorporate additional tuning parameters.
} 